\title{
CONTINUUM NEIGHBORHOODS AND FILTERBASES
}

\author{
DAVID P. BELLAMY ${ }^{1}$ AND HARVEY S. DAVIS ${ }^{2}$
}

Abstract. In this paper we prove that if $\Gamma$ is a filterbase of closed subsets of a compact Hausdorff space then $T(\cap \Gamma)$ $=\bigcap\{T(G) \mid G \in \Gamma\}$, where $T(A)$ denotes the set of those points for which every neighborhood which is a continuum intersects $A$ nonvoidly.

Introduction. In this paper $S$ denotes a compact Hausdorff space. If $p \in S$ and $W \subset S$, then $W$ is a continuum neighborhood of $p$ iff $W$ is a subcontinuum of $S$ and $p \in \operatorname{Int}(W)$. If $A \subset S, T(A)$ denotes the complement of the set of those points $p$ of $S$ for which there exists a continuum neighborhood which is disjoint from $A$ [1]. $S$ is said to be $T$-additive iff for every collection $\Lambda$ of closed subsets of $S$ whose union is closed, $T(\mathrm{U} \Lambda)=U\{T(L) \mid L \in \Lambda\}[2]$. The following three theorems are established.

TheOREM A. Let $\Gamma$ be a filterbase of closed subsets of $S$. Then $T(\cap \Gamma)$ $=\cap\{T(G) \mid G \in \Gamma\}$.

THEOREM B. $S$ is T-additive iff for each pair $A, B$ of closed subsets of $S, T(A \cup B)=T(A) \cup T(B)$.

TheOREM C. Let $A$ be a closed subset of $S$. If $K$ is a component of $T(A)$ then $T(A \cap K)=K \cup T(\varnothing)$.

Theorem A is used in establishing Theorems B and C. Theorem C is used to obtain the known result that if $S$ and $W$ are continua and $W \subset S$ then $T(W)$ is a continuum [1].

Proof of Theorem A. It is immediate from the definition that whenever $A \subset B, T(A) \subset T(B)$ and thus $T(\cap \Gamma) \subset \cap\{T(G) \mid G \in \Gamma\}$.

Suppose $p \notin T(\cap \Gamma)$. There exists $W$, a subcontinuum of $S$, such that $p \in \operatorname{Int}(W)$ and $W \cap(\cap \Gamma)=\varnothing$. Since $W$ is compact, there exists a finite collection $G_{1}, \cdots, G_{n}$ of elements of $\Gamma$ whose intersection is disjoint from $W$. By hypothesis there exists $G$, an element of $\Gamma$, which is contained in $G_{1} \cap \ldots \cap G_{n}$. Since $G$ is disjoint from $W, p \notin T(G)$. Hence $p \notin \cap\{T(G) \mid G \in \Gamma\}$ and thus

Received by the editors January 6, 1970.

AMS 1969 subject classifications. Primary 5455; Secondary 5465.

Key words and phrases. Compact Hausdorff space, continuum neighborhood, $T(A), T$-additive, filterbase, component.

1 Research supported in part by the University of Delaware Research Foundation.

${ }^{2}$ Research supported in part by the National Science Foundation NSF 71-1550. 


$$
T(\cap \Gamma)=\bigcap\{T(G) \mid G \in \Gamma) .
$$

Proof of Theorem B. The necessity of the condition is clear. Let $\Lambda$ be a collection of closed subsets of $S$ whose union is closed in $S$. Since $T(\cup \Lambda) \supset \cup\{T(L) \mid L \in \Lambda\}$, it need only be shown that $T(\mathrm{U} \Lambda)$ $\subset \cup\{T(L) \mid L \in \Lambda\}$ in order to establish the sufficiency of the condition.

Suppose $x \notin \cup\{T(L) \mid L \in \Lambda\}$. Then for each $L \in \Lambda$ let $F(L)$ be the collection of closed subsets $A$ of $S$ such that $L \subset \operatorname{Int}(A)$. If $L=\varnothing$, clearly $T(L)=\bigcap\{T(A) \mid A \in F(L)\}$. If $L \neq \varnothing$, then $F(L)$ is a filterbase of closed subsets of $S$ and, since $\cap F(L)=L, T(L)$ $=\cap\{T(A) \mid A \in F(L)\}$ by Theorem A.

Hence, for each $L, x \notin \cap\{T(A) \mid A \in F(L)\}$ and thus there exists, for each $L, f(L) \in F(L)$, such that $x \notin T(f(L))$. $\{\operatorname{Int}(f(L)) \mid L \in \Lambda\}$ is an open covering of $U \Lambda$. Since $U \Lambda$ is compact there exists a finite subcollection $\Gamma$ of $\{f(L) \mid L \in \Lambda\}$ such that $U \Lambda \subset U \Gamma$. Since, by hypothesis and induction $T(\cup \Gamma)=\bigcup\{T(G) \mid G \in \Gamma\}, \quad T(\mathrm{U} \Lambda)$ $\subset \cup\{T(G) \mid G \in \Gamma\}$. Since for all $G \in \Gamma, x \notin T(G)$, it follows that $x \notin T(\cup \Lambda)$. Thus $T(\cup \Lambda) \subset \cup\{T(L) \mid L \in \Lambda\}$.

Proof of Theorem C. Two technical lemmas are established. Theorem $\mathrm{C}$ follows easily from these two lemmas and Theorem A.

Lemma 1. Let $A$ be a subset of $S$. $p \in S-T(A)$ iff there is a subcontinuum $W$ and an open subset $Q$ of $S$ such that $p \in \operatorname{Int}(W) \cap Q, \operatorname{Fr}(Q)$ $\cap T(A)=\varnothing$ and $W \cap A \cap Q=\varnothing$.

Proof. Let $p \in S-T(A)$. There is a subcontinuum $W$ of $S$ such that $p \in \operatorname{Int}(W)$ and $W \cap A=\varnothing$. Since $S$ is regular there is an open subset $Q$ of $S$ such that $p \in Q$ and $\operatorname{Cl}(Q) \subset \operatorname{Int}(W)$. It is clear that $\operatorname{Fr}(Q) \cap T(A)=\varnothing$ and $W \cap A \cap Q=\varnothing$.

Now suppose that there is a subcontinuum $W$ and an open subset $Q$ of $S$ such that $p \in \operatorname{Int}(W) \cap Q, \operatorname{Fr}(Q) \cap T(A)=\varnothing$ and $W \cap A \cap Q$ $=\varnothing$. Since $\operatorname{Fr}(Q)$ is compact and disjoint from $T(A)$, there exists a finite collection $\left\{W_{i}\right\}$ of subcontinua of $S$, all disjoint from $A$, such that $U\left\{\operatorname{Int}\left(W_{i}\right)\right\} \supset \operatorname{Fr}(Q)$. Since if $W \subset Q$ it is immediate that $p \in S-T(A)$, assume $W \cap S-Q \neq \varnothing$. The closure of each component of $W \cap Q$ must intersect at least one of the $W_{i}$ 's, since $\operatorname{Fr}(Q) \subset U\left\{W_{i}\right\}$. Hence $(W \cap Q) \cup\left(\cup\left\{W_{i}\right\}\right)=H$ has only a finite number of components. Since $p \in \operatorname{Int}(W) \cap Q$, there is a component $K$ of $H$ such that $p \in \operatorname{Int}(K)$ and, of course, $K \cap A \subset H \cap A=\varnothing$. Thus $p \in S-T(A)$.

Lemma 2. Let $A$ be a subset of $S$. If $T(A)=M \cup N$ separate then $T(A \cap M)=M \cup T(\varnothing)$. 
Proof. Suppose $p \in T(A \cap M)-(M \cup T(\varnothing))$. Since $p \notin T(\varnothing)$, there is a subcontinuum $W$ of $S$ such that $p \in \operatorname{Int}(W)$. Since $S$ is normal, there is an open subset $Q$ of $S$ containing $N$ whose closure is disjoint from $M$. It is clear that $p \in \operatorname{Int}(W) \cap Q, \operatorname{Fr}(Q) \cap T(A \cap M)$ $\subset \operatorname{Fr}(Q) \cap T(A)=\varnothing$ and $W \cap(A \cap M) \cap Q \subset Q \cap M=\varnothing$. Hence, by Lemma 1, $p \notin T(A \cap M)$, thus contradicting the supposition.

Now suppose that $p \in(M \cup T(\varnothing))-T(A \cap M)$. Since $p \notin T(A \cap M)$ and $\varnothing \subset A \cap M, p \notin T(\varnothing)$. Hence $p \in M$. There is an open subset $Q$ of $S$ containing $M$ whose closure is disjoint from $N$. Since $p \notin$ $T(A \cap M)$, there is a subcontinuum $W$ of $S$ such that $p \in \operatorname{Int}(W)$ and $W \cap(A \cap M)=\varnothing$. It is clear that $p \in \operatorname{Int}(W) \cap Q$ and $\operatorname{Fr}(Q)$ $\cap T(A)=\varnothing$. Since $Q \cap N=\varnothing, W \cap A \cap Q=W \cap(A \cap M)=\varnothing$. Hence, by Lemma $1, p \notin T(A)$ so $p \notin M$, thus contradicting the supposition.

Now in order to establish Theorem $\mathrm{C}$, let $A$ be a closed subset of $S$ and $K$ be a component of $T(A)$. Let $\left\{K_{\alpha}\right\}$ be the collection of all subsets of $T(A)$ such that $K \subset K_{\alpha}$ and $K_{\alpha}$ is both open and closed in $T(A)$. Note that the collection $\left\{A \cap K_{\alpha}\right\}$ can only fail to be a filterbase if for some $K_{\alpha}, A \cap K_{\alpha}=\varnothing$. In this case the conclusion of Theorem $\mathrm{A}$ is trivial. Lemma 2 , of course, remains true even if $A \cap M=\varnothing$ so, for each $K_{\alpha}, T\left(A \cap K_{\alpha}\right)=K_{\alpha} \cup T(\varnothing)$. That this can occur is seen by letting $S$ be the Cantor set, $A$ be the void set and $K_{\alpha}$ be $S$.

The following sequence of equalities establish the theorem:

$$
\begin{aligned}
T(A \cap K) & =T\left(\cap\left\{A \cap K_{\alpha}\right\}\right)=\cap\left\{T\left(A \cap K_{\alpha}\right)\right\} \\
& =\bigcap\left\{K_{\alpha} \cup T(\varnothing)\right\}=\bigcap\left\{K_{\alpha}\right\} \cup T(\varnothing) \\
& =K \cup T(\varnothing) .
\end{aligned}
$$

Theorem $\mathrm{C}$ is not true if the requirement that $A$ be closed is dropped. Let $S$ be the unit interval and let $A$ be the sequence $\{1 / n\}$. Then $T(A)=\{0\} \cup A$. Let $K=\{0\}$. Then $T(A \cap K)=T(\varnothing)$ which is void since $S$ is a continuum. But $K \cup T(\varnothing)$ is not void.

Corollary 1. Let $S$ be a continuum and $W$ be a subcontinuum of $S$. $T(W)$ is a subcontinuum of $S$.

Proof. Suppose $T(W)=A \cup B$ separate. By Theorem $C, T(W \cap A)$ $=A$ and $T(W \cap B)=B$ since $T(\varnothing)=\varnothing$ when $S$ is a continuum. $W \cap A \neq \varnothing$ since $T(W \cap A) \neq \varnothing$ and, likewise $W \cap B \neq \varnothing$. Hence $W=(W \cap A) \cup(W \cap B)$ separate, contradicting the hypothesis and thus establishing the proposition. 
Corollary 2. Let $S$ be a continuum and let $W_{1}$ and $W_{2}$ be subcontinua of $S$. If $T\left(W_{1} \cup W_{2}\right) \neq T\left(W_{1}\right) \cup T\left(W_{2}\right)$ then $T\left(W_{1} \cup W_{2}\right)$ is a continuum.

Proof. Suppose $T\left(W_{1} \cup W_{2}\right)=A \cup B$ separate. By Lemma 2, $T\left(\left(W_{1} \cup W_{2}\right) \cap A\right)=A$ and $T\left(\left(W_{1} \cup W_{2}\right) \cap B\right)=B$. Suppose $W_{1} \subset A$. If $W_{2} \subset A$ then $A=T\left(\left(W_{1} \cup W_{2}\right) \cap A\right)=T\left(W_{1} \cup W_{2}\right)$, thus contradicting the supposition. Hence $W_{2} \subset B$. But then $T\left(W_{1}\right)=A$ and $T\left(W_{2}\right)$ $=B$. Thus $T\left(W_{1} \cup W_{2}\right)=T\left(W_{1}\right) \cup T\left(W_{2}\right)$. Corollaries 1 and 2 are special cases of Theorem 8 of [1].

Corollary 3. Let $S$ be a continuum and let $A$ and $B$ be closed subsets of $S$. If $K$ is a component of $T(A \cup B)$ which lies in neither $T(A)$ nor $T(B)$, then, $K \cap A \neq \varnothing \neq K \cap B$.

Proof. Since $S$ is a continuum, $T(\varnothing)=\varnothing$ and, by Theorem $\mathrm{C}$, $T((A \cup B) \cap K)=K$. Since $K$ lies in neither $T(A)$ nor $T(B),(A \cup B)$ $\cap K$ meets both $A$ and $B$. Thus $K$ meets both $A$ and $B$.

Corollary 4. Let $S$ be a continuum and let $A$ and $B$ be closed subsets of $S$. If $T(A \cup B) \neq T(A) \cup T(B)$ then there exists a subcontinuum $K \subset T(A \cup B)$ such that $K \cap A \neq \varnothing \neq K \cap B$.

Proof. Let $K$ be the component of some point in $T(A \cup B)$ $-(T(A) \cup T(B))$ and apply Corollary 3.

\section{BIBLIOGRAPHY}

1. H. S. Davis, D. P. Stadtlander and P. M. Swingle, Properties of the set functions $T^{n}$, Portugal. Math. 21 (1962), 113-133. MR 25 \#5501.

2. H. S. Davis, A note on connectedness in kleinen, Proc. Amer. Math. Soc. 19 (1968), 1237-1241.

Michigan State University, East Lansing, Michigan 48823

University of Delaware, Newark, Delaware 19711 